\title{
Long-term Perseveration in Alzheimer's Disease: A Case Report
}

\author{
EDITH V. SULLIVAN
}

Department of Cognitive and Brain Sciences and Clinical Research Center, Massachusetts Institute of Technology, and Department of Neurology, Massachusetts General Hospital, Massachusetts, USA

Current address: Department of Psychiatry and Brain Sciences, Stanford University School of Medicine, Stanford, CA 94305, USA, and Psychiatry Service (116A3), Department of Veterans Affairs Medical Center, 3801 Miranda Avenue, Palo Alto, CA 94304, USA

\begin{abstract}
The most common clinical sign of Alzheimer's disease (AD) is progressive memory loss. Presented here is a case of $\mathrm{AD}$ who, despite ultimate profound dementia with severe amnesia, showed retention of a perseverative response she developed during 26 encounters, over 4.5 years, with the Brown-Peterson distractor test. From Test 9 onwards, she responded from the first distractor-filled trial with one consonant trigram, appearing in none of the seven test forms given her. At Test 26, she could not repeat heard trigrams yet faithfully responded with her perseverative trigram. The trigram, ostensibly declarative information, apparently became part and parcel of the task's procedure. Although perseveration is a form of impairment probably resulting from Alzheimer pathology involving frontal and parietal cortex, it may also reflect a form of preserved memory, albeit distorted, supported by posterior cortical regions spared in $\mathrm{AD}$.
\end{abstract}

\section{Introduction}

The most common clinical sign of Alzheimer's disease (AD) is progressive memory loss. In rigorous tests of memory, poor scores of $\mathrm{AD}$ patients are fraught with omissions and perhaps more interestingly with intrusions and perseverations (Fuld et al., 1982). Perseveration refers to a response, which had been elicited to an earlier-occurring stimulus but is inappropriately elicited again to a different, later-occurring stimulus. The occurrence of intrusive and perseverative errors implies that information is not entirely forgotten, but rather that it is retained in part or whole but reused incorrectly. This observation suggests that the contextual cues required to evoke an answer to a new, specific situation are ignored. Perseveration may represent a special form of memory not fully considered by prevailing theories of memory. Presented here is a case of AD who developed an incorrect, perseverative response with repeated exposure to a memory test over a period of months and used it during testing for years.

\section{Case Report}

History. The patient was enrolled in a longitudinal study, which incorporated a series of drug treatment studies, at the Clinical Research Center of the Massachu- 


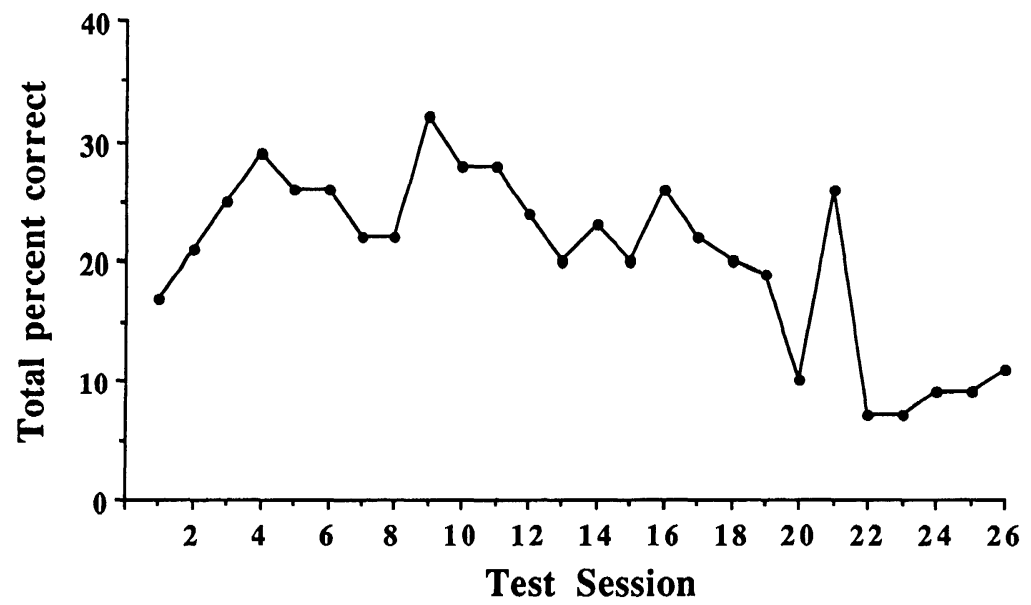

FIG. 1.

setts Institute of Technology. When first studied, she was 56 years old, had symptoms of $\mathrm{AD}$ for about 2 years, and was mildly demented as assessed by clinical criteria (Corkin, 1982). Her initial symptoms included memory loss and spatial disorientation. She had 12 years of education and was right handed. She died at age 65. A CT scan revealed marked cortical atrophy, enlarged ventricles, and prominent Sylvian fissures.

Procedure. During her many test sessions over the course of 4.5 years, she was administered the Brown-Peterson distractor test of short-term memory (STM) 26 times (Brown, 1958; Peterson and Peterson, 1959). She received the STM test under a variety of interoceptive and exteroceptive conditional changes: she was tested by five examiners, in four testing rooms, with seven forms of the test, under six drug states (lecithin, oral and i.v. physostigmine, tryptophan, and hydergine and their respective placebos), with six retention intervals, and during progressive stages of the disease (mild to profound).

Each STM test session comprised at least three practice trials and 24 test trials, with four trials at each of six retention intervals. The tests were run in blocks of increasing length: $0,3,6,9,15$, and $30 \mathrm{sec}$. The memory items were consonant trigrams prerecorded on tape. For each trial, the patient heard a trigram, repeated it, heard a three-digit number, repeated it and subtracted 3 or 1 from it, depending upon her current ability. After a retention interval, the patient attempted to recall the trigram from that trial and was encouraged to guess; $10 \mathrm{sec}$ was allotted for responding. Trials were separated by $15 \mathrm{sec}$ in order to minimize proactive interference (Kincaid and Wickens, 1970).

Results. On all 26 STM tests the AD patient was impaired. She completed the test on all occasions, except the third because of nausea and vomiting resulting from an infusion of physostigmine $(0.5 \mathrm{mg})$. She showed slight fluctuations in scores over the first 19 tests, followed by a consistent drop over the last five tests (Fig. 1). From Test 1 to 9, forgetting fell to asymptote at the 3- or 6-sec retention interval; thereafter, the asymptote generally occurred at the 3 -sec interval. By contrast, healthy elderly subjects reach asymptote at 9 sec (Corkin, 1982; Sullivan et al., 1986). 
The first time the AD patient produced her perseverative response, "KLX," was on Trial 9 of Test 3 , which was 1.5 months after her first test. This trigram had never appeared as a test item nor was it presented in any of the test forms she received subsequently. From Test 9 to 21 , which occurred over the course of 2 years and 10 months, she responded with this trigram on virtually every trial, except for trials at the 0-sec retention interval; importantly, at this interval, her responses approximated or replicated the given test trigrams. Furthermore, perseverative recall persisted even though this patient accurately repeated the trigrams upon hearing them. In the last five tests, however, she was so aphasic that she could not repeat the test trigrams correctly. Despite this disability in language production, she responded exclusively and without dysphasia with the perseverative trigram, KLX. She herself usually expressed enthusiasm for going this particular test of the battery because she felt she knew how to do it. Examples of her responses appear in Table 1.

\section{Discussion}

This patient seemed to rely upon her perseverative response at times when her recall of trigrams was difficult: at long retention intervals and when she was no longer able to repeat given test items. Once perseveration started reliably, she virtually never made errors of omission even though she had done so, particularly at the long retention intervals, prior to Test 9. Moreover, her perseverative response abided by the rules of the task at hand: she always produced a response within the 10 -sec recall period and the response met the requirements that it contain three consonants.

The reliance upon perseverative responses when recall becomes difficult was noted previously by Shindler et al. (1984). They hypothesized that when longterm memory (LTM) failed, information available in STM would be used in responding. In the present case, the reverse seemed to be true: when STM or working memory failed, stable information from some form of LTM store was retrieved.

In seeking reasons why the perseverative response was "KLX" specifically, it is important to consider the practice trial trigram, "LCK," which was given at each test session. The practice trigram and the patient's perseverative trigram shared two letters but in reversed order and with an intervening letter. Repeated exposure to the practice trigram may have enhanced trace strength for those letters and thus may have acted as a priming agent. It is curious, however, that the only time she would use the perseverative trigram was during the recall interval, and never during the repetition interval; thus, her response was not merely an automatism (cf. Fuld et al., 1982). Alternatively, one could argue that this patient did not understand that she was to recall the just given (and newly repeated) trigram during the retention interval. Performance at the 0 -sec retention interval, however, provided evidence that she did understand this aspect of the test because she typically recalled the exact trigram or a close approximation at this interval for the first 19 test sessions (see Table 1 for such instances).

In addition to the one case tested over 4.5 years, two additional AD cases had 
TABLE 1. Examples of the AD patient's responses on the STM distractor test over 4.5 years

\begin{tabular}{|c|c|c|c|c|c|c|c|c|c|}
\hline \multicolumn{5}{|c|}{ Test $l$} & \multicolumn{5}{|c|}{ Test 9} \\
\hline Retention interval & Test trigram & Subject's repeat & Recall & Score & Retention interval & Test trigram & Subject's repeat & Recall & Score \\
\hline \multirow[t]{5}{*}{0 seconds } & & & & & 0 seconds & & & & \\
\hline & $\mathrm{XGC}$ & $\mathrm{XGC}$ & $\mathrm{XGC}$ & 4 & & $\mathrm{XGC}$ & $\mathrm{XGZ}$ & XZG & 4 \\
\hline & PTM & PTM & PTM & 6 & & PTM & PTM & PTM & 6 \\
\hline & KBQ & $\mathrm{KBQ}$ & P- & 0 & & $\mathrm{KBQ}$ & $\mathrm{KBQ}$ & $\mathrm{KBQ}$ & 6 \\
\hline & FLS & FLS & FLS & 6 & & FLS & FLS & FL.S & 6 \\
\hline \multirow[t]{5}{*}{3 seconds } & & & & & 3 seconds & & & & \\
\hline & BKD & BKD & - & 0 & & BKD & BKD & BKD & 6 \\
\hline & SFH & SFH & - & 0 & & SFH & SFH & SKF & 3 \\
\hline & BNR & BNR & - & 0 & & BNR & BNR & $\mathrm{KNX}$ & 2 \\
\hline & MJZ & MJZ & MJ- & 4 & & MJZ & MJZ & KMX & 1 \\
\hline \multirow{5}{*}{6 seconds } & & & & & 6 seconds & & & & \\
\hline & HPL & HPL & - & 0 & & HPL & HPL & KLM & 1 \\
\hline & XBM & XBM & BMX & 3 & & $\mathrm{XBM}$ & XBM & LXM & 3 \\
\hline & JQK & $\mathrm{JQK}$ & KXM & 1 & & $\mathrm{JQK}$ & $\mathrm{JQK}$ & KLX & 1 \\
\hline & FTC & FTC & $\mathrm{XPM}$ & 0 & & FTC & FTC & KLX & 0 \\
\hline \multirow{5}{*}{9 seconds } & & & & & 9 seconds & & & & \\
\hline & DSJ & DSJ & - & ) & & DSJ & DSJ & KLX & 0 \\
\hline & $\mathrm{ZQL}$ & $\mathrm{ZQL}$ & $\mathrm{XZ}$ - & 1 & & ZQL & $\mathrm{ZQL}$ & KLX & 1 \\
\hline & RHB & RHB & $\mathrm{X}-$ & 0 & & RHB & RHB & KLX & 0 \\
\hline & GPX & GPX & - & 0 & & GPX & GPX & $\mathrm{KLX}$ & 2 \\
\hline \multirow[t]{5}{*}{15 seconds } & & & & & 15 seconds & & & & \\
\hline & FNB & FNV & - & 0 & & FNB & FNB & $\mathrm{KLX}$ & 0 \\
\hline & RMC & RMC & - & 0 & & RMC & RMC & $\mathrm{KLX}$ & 0 \\
\hline & GJV & GJV & - & 0 & & GJV & GJV & KLX & 0 \\
\hline & DQS & DQS & $\cdots$ & 0 & & DQS & DQS & $\mathrm{KLX}$ & 0 \\
\hline \multirow[t]{6}{*}{30 seconds } & & & & & 30 seconds & & & & \\
\hline & LDX & LDX & - & 0 & & LDX & LDX & KLX & 3 \\
\hline & BKT & BKT & - & 0 & & BKT & BKT & KLX & 1 \\
\hline & $\mathrm{NZP}$ & NZP & - & 0 & & NZP & NZP & KLX & 0 \\
\hline & HRG & HRG & - & 0 & & HRG & HRG & KLX & 0 \\
\hline & & & \multicolumn{2}{|c|}{ Total $=25$} & & & & \multicolumn{2}{|c|}{ Total $=46$} \\
\hline \multicolumn{5}{|c|}{ Test 16} & & & est 26 & & \\
\hline Retention interval & Test trigram & Subject's repeat & Recall & Score & Retention interval & Test trigram & Subject's repeat & Recall & Score \\
\hline (0) seconds & & & & & 0 seconds & & & & \\
\hline & QSD & QSD & QSD & 6 & & MCX & $\mathrm{M}-$ & - & 0 \\
\hline & BKV & BKB & BKC & 4 & & FHR & $\mathrm{F}-$ & - & 0 \\
\hline & GFN & GFN & GFN & 6 & & LJT & $\mathrm{K}-$ & - & 0 \\
\hline & FCJ & $\mathrm{FCJ}$ & FCJ & 6 & & PKD & key- & KLX & 1 \\
\hline 3 seconds & & & & & 3 seconds & & & & \\
\hline & CLR & CLR & $\mathrm{KLX}$ & 2 & & GTK & G- & $\mathrm{KLX}$ & 1 \\
\hline & HMK & HMK & $\mathrm{KLX}$ & 1 & & JPC & JKkey & dayKL & 0 \\
\hline & BSZ & BSZ & BLX & 2 & & $\mathrm{BHZ}$ & Bzay & KLX & 0 \\
\hline & XPD & XPD & KLX & 1 & & QMF & $\mathrm{K}-$ & KLX & 1 \\
\hline 6 seconds & & & & & 6 seconds & & & & \\
\hline & JXF & JXF & $\mathrm{KLX}$ & 1 & & VRL & - & KLX & 1 \\
\hline & DMS & DMS & $\mathrm{KLX}$ & 0 & & SFQ & - & $\cdots$ & 0 \\
\hline & HGL & HGL & KL.X & 1 & & $\mathrm{NZG}$ & - & KLX & 0 \\
\hline & $\mathrm{KNC}$ & $\mathrm{KNC}$ & KLX & 2 & & DXK & B-- & KLX & 2 \\
\hline 9 seconds & & & & & 9 seconds & & & & \\
\hline & BTL & BTL & $\mathrm{KLX}$ & 1 & & HLS & ay & KLX & 2 \\
\hline & JQZ & $\mathrm{JQZ}$ & KLX & 0 & & QDR & Q- & KLX & 0 \\
\hline & $\mathrm{XMH}$ & $\mathrm{XMH}$ & $\mathrm{KLX}$ & 1 & & PCM & $\mathrm{K}-$ & KLX & 0 \\
\hline & FVR & FVR & $\mathrm{KLX}$ & 0 & & VNG & B- & $\mathrm{KLX}$ & 0 \\
\hline 15 seconds & & & & & 15 seconds & & & & \\
\hline & SLG & SLG & $\mathrm{KLX}$ & 2 & & NJT & - & KLX & 0 \\
\hline & $\mathrm{TCN}$ & $\mathrm{TCN}$ & KLX & 0 & & $\mathrm{BRZ}$ & B- & KLX & 0 \\
\hline & RPK & RPK & KLX & 1 & & SPV & - & KLX & 0 \\
\hline & VHQ & BHQ & $\mathrm{KLX}$ & 0 & & $\mathrm{XFQ}$ & - & KLX & 1 \\
\hline 30 seconds & & & & & 30) seconds & & & & \\
\hline & DJT & DJT & $\mathrm{KL}$.X & 0 & & KXB & KLX & KLX & 6 \\
\hline & PRF & PRF & $\mathrm{KLX}$ & 0 & & GSM & - & KLX & 0 \\
\hline & BXQ & BXQ & KLX & 1 & & THL & - & $\mathrm{KLX}$ & 1 \\
\hline & ZGM & ZGM & KLX & 0 & & $\mathrm{ZCJ}$ & - & $\mathrm{KLX}$ & 0 \\
\hline & & & Total $=$ & & & & & Total $=$ & 16 \\
\hline
\end{tabular}


taken the STM test on multiple occasions: 19 and 22 times over a period of 16 and 21 months. These patients were similar to the featured case by spontaneously creating incorrect, unique perseverative responses that abided by the rules of the test. These two patients differed from the case presented, however, in that they did not carry over a specific trigram to subsequent testing sessions. Thus, their perseverative responses were of the type commonly reported in tests of memory (e.g. Fuld, et al., 1982).

Within the taxonomy of perseveration offered by Sandson and Albert (1984), the type of perseveration most suitable to that described here is recurrent, "... the unintentional repetition of a previous response to a subsequent stimulus, involv[ing] an abnormal post-facilitation of memory traces ..." (p. 715). Within Liepmann's classification (1905; reviewed in Sandson and Albert, 1984), it is called intentional and refers to motor responses previously performed and reenacted when a different response was intended. On two accounts, this definition does not entirely fit this case. Firstly, there is reason to believe that the patient's responses were not unintentional because she never showed insight into the repetitive nature of her response; by contrast, the other two AD patients did reveal insight into their perseverations, thus suggesting that they were incorrect as well as unintentional. For example, one patient spontaneously offered the following observations about his perseverative responses in three different test sessions: "Now I forget 'JQR' [i.e. the perseverative response] and rest"; "CJP [i.e. the perseverative response], are we still on this?"; and after perseverating on "QST" said several times that "QST" was wrong but that it was his best answer. Secondly, the type of memory traces overly facilitated during perseveration are highly specific and may qualify as fact, declarative, or episodic memory. Fact memory, however, is severely compromised in patients with $\mathrm{AD}$, as it was in the presented case. Consequently, it is difficult to attribute perfect recall, observed in her perseverative response, to an intact fact memory system.

Perseveration can be considered to have a dual character: it is incorrect yet consistent. In an attempt to speculate about the neural basis for perseveration, considered here are compromised brain systems, which may contribute to the production of aberrant responses, and spared brain systems, which may underlie consistent performance. Perseveration has been attributed to poor capacity to use contextual cues, such that new and old stimuli and responses lose their temporal or spatial tags (Buckingham et al., 1981). Temporal contextual memory, as measured by recency discrimination, is impaired in AD (Sagar et al., 1988; Sullivan and Sagar, 1989) and accordingly may contribute to perseveration. That temporal contextual discrimination is also impaired in patients with frontal-lobe lesions but spared in patients with medial temporal-lobe lesions (Milner, 1971; Sagar $e t$ al., 1990; Schacter, 1987; Smith and Milner, 1983, 1988) implicates frontal-lobe pathology in perseveration. More direct evidence for a frontal-lobe basis of perseveration abounds in the neuropsychological literature; for example, a high rate of perseverative errors on the Wisconsin Card Sorting Test occurs in patients with lesions invading the dorsolateral prefrontal cortex (Milner, 1964). An important area of additional pathology is probably the hippocampus, which is a common site of dense Alzheimer pathology (e.g. Blessed et al., 1968; Kemper, 1984). While memory test performance of patients with unilateral or bilateral hippocam- 
pal pathology is not marked by perseveration, hippocampal and frontal-lobe pathology may provide a critical combination of loss of contextual cues as well as poor recall of past events or responses to produce severe perseveration, which may entail forgetting that a perseverative response has already been used. A third cortical site, the posterior parietal region, may also contribute to recurrent perseveration. Sandson and Albert (1984) reported verbal recurrent perseveration in a patient with a speech disorder and right-sided weakness who had a left hemisphere infarction, and nonverbal recurrent perseveration in a patient with rightsided weakness and mutism who had a left fronto-parietal infarction. In a study by Freedman and Oscar-Berman (1987), AD patients committed significantly more perseverative errors than did demented patients with PD in a tactile reversal of original learning task, which provides a measure of perseveration; the $\mathrm{AD}$ deficit was attributed to lesions of the parietal system. Invoking both the dorsolateral prefrontal and posterior parietal cortices as contributing to perseveration is reasonable considering the multiple anatomical connections between these sites documented in monkey (Selemon and Goldman-Rakic, 1988). Thus, the anterior three-quarters of the cortex and medial temporal regions of the brain may be implicated in producing long-term, recurrent perseveration. Indeed AD is commonly marked by pathology in these regions (e.g. Brun and Englund, 1981; Kemper, 1984).

Cortical regions spared in AD include feed-forward areas of the occipital lobes and primary motor cortex (e.g. Brun and Englund, 1981; Kemper, 1984). Extrastiate visual cortex has been hypothesized to subserve, at least in part, the spared learning capacity of repetition priming for visually-presented information in AD (Gabrieli et al., 1991). The role of spared motor cortex has not yet been explored in AD memory research. Priming refers to response facilitation to specific stimuli previously experienced and involves implicit, or unconscious, rather than explicit memory processes (e.g., Gabrieli, 1990; Graf et al., 1984; McAndrews et al., 1984; Tulving and Schacter, 1990; Warrington and Weiskrantz, 1974); it does not merely reflect reactivation of preexisting memories, i.e. premorbidly-encoded semantic information Smith and Oscar-Berman, 1990; Tulving and Schacter, 1990). According to Tulving and Schacter (1990), priming is independent of explicit memory and is "hyperspecific." Clearly the robustness of the perseverative response described here contrasts with the patient's severely impaired explicit memory; furthermore, her perseverative response was linked exclusively to a specific, meaningless trigram retrieved within a specific set of constraints (i.e. the rules of the STM test).

In addition to priming, the perseveration noted here may reflect a form of procedural, or ruled-based, learning because the responses always fell within the rules of the test (Cohen and Squire, 1980). Because the stimuli used in the STM test were auditory and demanded articulated responses, spared capacity to perform this test within a given rule set may be linked to an auditory/articulatory cortical system analogous to the visual system proposed for preserved priming in the visual modality. As with the proposed visual mnemonic system spared in AD, such a system would be presumed to operate presemantically and pre-linguistically. Thus, this system could support preservation of articulation for the primed trigram despite disability in phoneme articulation. Supporting this position is a 
report by Morris (1984) demonstrating that the articulatory loop system is intact in $\mathrm{AD}$. This system is a principal component of working memory (Baddeley, 1987) and is defined as "a buffer store of limited duration fed either phonetically or by articulation" (Morris, 1984, p. 144). Such systems may further provide a theoretical basis as to why word reading, as done in the National Adult Reading Test (NART; Nelson and O'Connell, 1978), remains relatively preserved and provides a good estimate of premorbid intelligence through moderate to severe dementia (Stebbins et al., 1990).

The long-term, recurrent perseveration of the $\mathrm{AD}$ patient presented here provides evidence for two dissociations: impaired recall with spared, rule-driven performance, and impaired spontaneous and repetitive language production with spared production of a perseverative consonant trigram. The impairment in the form of perseveration may be attributable to pathology of the much of the anterior cortex and medial temporal lobes, and the preservation of performance in the form of perseverative regularity may be attributable to the sparing of primary motor and extrastriate cortices in AD. These observations suggest that despite severe memory impairment for newly-presented facts, the capacity to formulate and remember a strategy that abides by rule constraints can persist in AD. This preservation of capacity to use rules, even though deplete of flexibility and usefulness within normal standards, provides some indication that perseveration, perhaps like rituals and obsessions documented in patients with frontal-lobe pathology, represents an attempt to override the loss of ability to process, use, and remember episodic information. Perseveration, then, might be considered the misuse and misinterpretation of episodic data in terms of procedural knowledge, that is, procedural learning gone awry.

\section{Acknowledgements}

I thank Suzanne Corkin, John H. Growdon, John D. E. Gabrieli, and Margaret M. Keane for thoughtful comments on this paper as well as the following people for their help in testing: Rae Ann Clegg, Kathleen Coffin, Allison Feeley, John D. E. Gabrieli, Margaret M. Keane, Marguerite Randolf, and Karen J. Shedlack. This research was supported by grants from the National Institute of Health to the Massachusetts Institute of Technology (RR 00088, MH 32724, and MH 2433) and to the Harvard Medical School at the Massachusetts General Hospital (P50-AG 05134). Support for E. V. Sullivan during manuscript preparation was provided by a grant from the National Institute of Health to the Stanford University School of Medicine (MH 40041) and by the Medical Research Service of the Department of Veterans Affairs.

\section{References}

Baddeley, A.D. (1987). "Working Memory." Oxford University Press, Oxford.

Blessed, G., Tomlinson, B.E. and Roth, M. (1968). The association between quantitative measures of dementia and of senile changes in the cerebral grey matter of elderly subjects. British Journal of Psychiatry, 114, 797-811. 
Brown, J. (1958). Some tests of the decay theory of immediate memory. Quarterly Journal of Experimental Psychology, 10, 12-21.

Brun, A. and Englund, E. (1981). Regional pattern of degeneration in Alzheimer's disease: neuronal loss and histopathological grading. Histopathology, 5, 549-564.

Buckingham, H., Whitaker, H. and Whitaker, H. (1981) On linguistic perseveration. In "Studies in Neurolinguistics" (Eds H. Whitaker and H. Whitaker). Academic Press, New York.

Cohen, N.J. and Squire, L.R. (1980). Preserved learning and retention of pattern-analyzing skill in amnesia: dissociation of knowing how and knowing that. Science, 210, 207-210.

Corkin, S. (1982). Some relationships between global amnesia and the memory impairments in Alzheimer's disease. In "Alzheimer's Disease: A Report of Progress in Research." (Eds S. Corkin, K. L. Davis, J. H. Growdon, E. Usdin and R. J. Wurtman), pp. 149-164. Raven Press, New York.

Freedman, M. and Oscar-Berman, M. (1987). Tactile discrimination learning deficits in Alzheimer's and Parkinson's diseases. Archives of Neurology, 44, 394-398.

Fuld, P.A., Katzman, R., Davies, P. and Terry, R.D. (1982). Intrusions as a sign of Alzheimer dementia: chemical and pathological verification. Annals of Neurology, 11, 155-159.

Gabrieli, J.D.E. (1990). Differential effects of aging and age-related neurological diseases on memory subsystems of the brain. In "The Handbook of Neuropsychology" (Eds F. Boller and J. Grafman), pp. 149-166. Elsevier, New York.

Gabrieli, J.D.E., Milberg, W., Keane, M.M. and Corkin, S. (1991). Intact priming of patterns despite impaired memory. Neuropsychologia, 28, 417-427.

Graf, P., Squire, L.R. and Mandler, G. (1984). The information that amnesic patients do not forget. Journal of experimental Psychology: Learning, Memory, and Cognition, 10, 164-178.

Kemper, T.L. (1984). Neuroanatomical and neuropathological changes in normal aging and dementia. In "Clinical Neurology of Aging" (Ed M.L. Albert), pp. 9-52. Oxford University Press, Oxford.

Kincaid, J.P. and Wickens, D.D. (1970). Temporal gradient of release from proactive inhibition. Journal of Experimental Psychology, 86, 313-316.

McAndrews, M.P., Glisky, E.L. and Schacter, D.L. (1987). When priming persists: long-lasting implicit memory for a single episode in amnesic patients. Neuropsychologia, 25, 497-506.

Milner, B. (1971). Interhemispheric differences in the localization of psychological processes in man. British Medical Bulletin, 27, 272-277.

Milner, B. (1964). Some effect of frontal lobotomy in man. In "The Frontal Granular Cortex and Behavior" (Eds J. Warren and K. Akert), pp. 313-334. McGraw-Hill, New York.

Morris, R. G. (1984). Dementia and the functioning of the articulatory loop system. Cognitive Neuropsychology, 1, 143-157.

Nelson, H.E. and O'Connell, A. (1978). Dementia: the estimation of premorbid intelligence levels using the New Adult Reading Test. Cortex, 14, 234-244.

Peterson, L.R. and Peterson, M.J. (1959). Short-term retention of individual items. Journal of Experimental Psychology, 58, 193-198.

Sagar, H.J., Gabrieli, J.D.E., Sullivan, E.V. and Corkin, S. (1990). Recency and frequency discrimination in the amnesic patient H.M. Brain, 113, 581-602.

Sagar, H.J., Sullivan, E.V., Gabrieli, J.D.E., Corkin, S. and Growdon, J.H. (1988) Temporal ordering and short-term memory deficits in Parkinson's disease. Brain, 111, 525-539.

Sandson, J. and Albert, M.L. (1984). Varieties of perseveration. Neuropsychologia, 22, 715-732.

Schacter, D.L. (1987). Memory, amnesia, and frontal lobe dysfunction. Psychobiology, 15, 21-36.

Selemon, L.D. and Goldman-Rakic, P.S. (1988). Common cortical and subcortical targets of the dorsolateral prefrontal and posterior parietal cortices in the Rhesus monkey: evidence for a distributed neural network subserving spatially guided behavior. The Journal of Neuroscience, 8, 4049-4068.

Shindler, A.G., Caplan, L.R. and Hier, D.B. (1984). Intrusions and perseverations, Brain and Language, 23, 148-158.

Smith, M.L. and Milner, B. (1983). Effects of focal brain lesions on sensitivity to frequency of occurrence. Society for Neuroscience Abstracts, 9, 30.

Smith, M.L. and Milner, B. (1988). Estimation of frequency of occurrence of abstract designs after frontal temporal lobectomy. Neuropsychologia, 26, 297-306. 
Smith, M.E. and Oscar-Berman, M. (1990). Repetition priming of words and pseudowords in divided attention and in amnesia. Journal of Experimental Psychology: Learning, Memory, and Cognition, 16, 1033-1042.

Stebbins, G.T., Wilson, R.S., Gilley, D.W., Bernard, B.A. and Fox, J.H. (1990). Use of the National Adult Reading Test to estimate premorbid IQ in dementia. The Clinical Neuropsychologist, $\mathbf{4}$, $18-24$.

Sullivan, E.V., Corkin, S. and Growdon, J.H. (1986). Verbal and nonverbal short-term memory in patients with Alzheimer's disease and in healthy elderly subjects. Developmental Neuropsychology, 2, 387-400.

Sullivan, E.V. and Sagar, H.J. (1989). Nonverbal recognition and recency discrimination deficits in Parkinson's disease and Alzheimer's disease. Brain, 112, 1503-1517.

Tulving, E. and Schacter, D.L. (1990). Priming and human memory systems. Science, 247, 301-306.

Warrington, E.K. and Weiskrantz, L. (1974). The effect of prior learning on subsequent retention in amnesic patients. Neuropsychologia, 12, 419-428. 


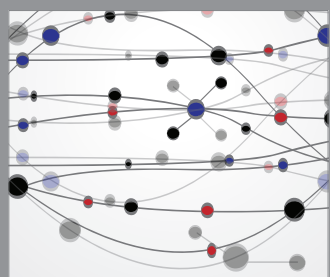

The Scientific World Journal
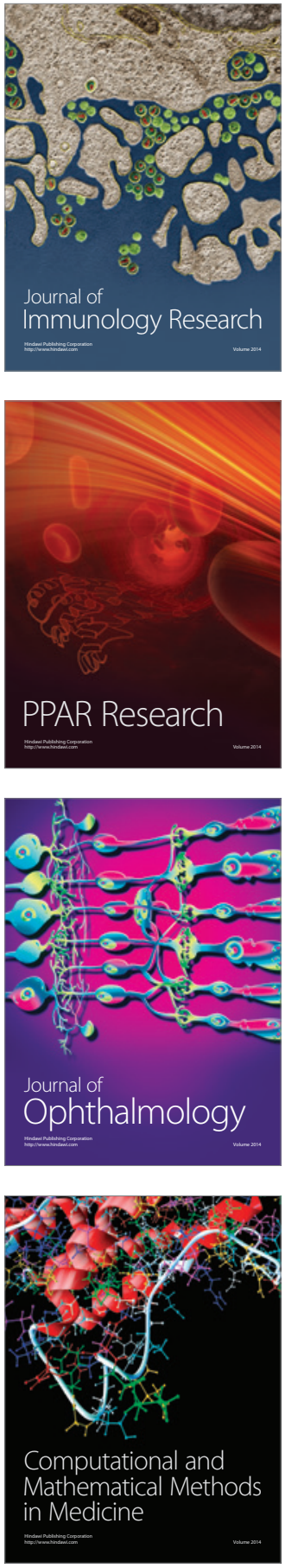

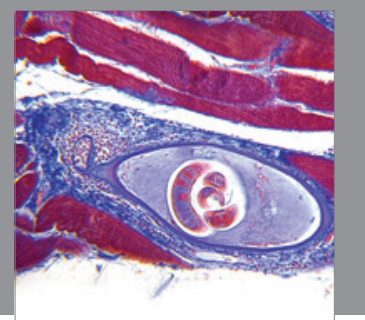

Gastroenterology

Research and Practice
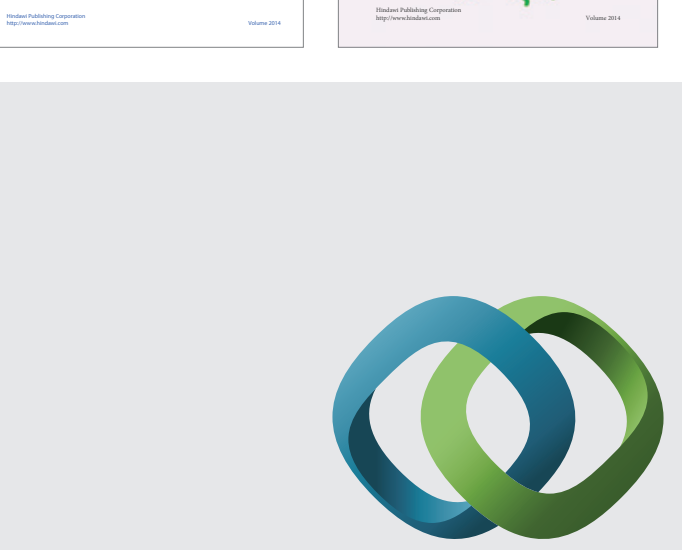

\section{Hindawi}

Submit your manuscripts at

http://www.hindawi.com
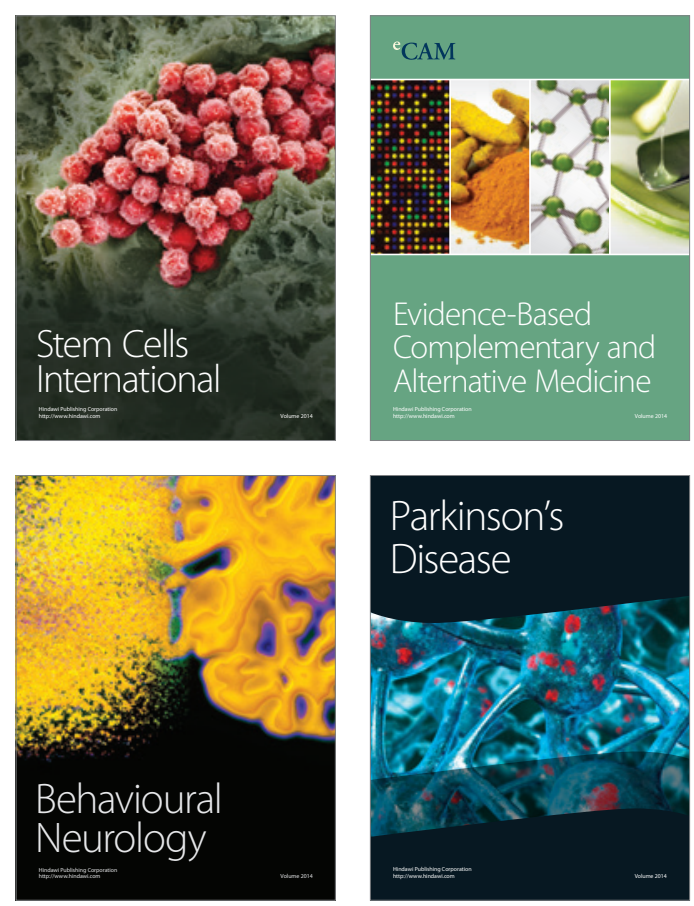

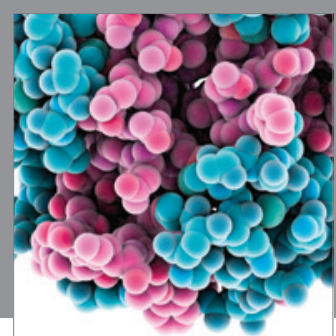

Journal of
Diabetes Research

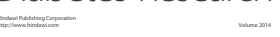

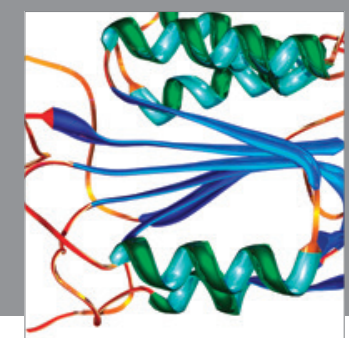

Disease Markers
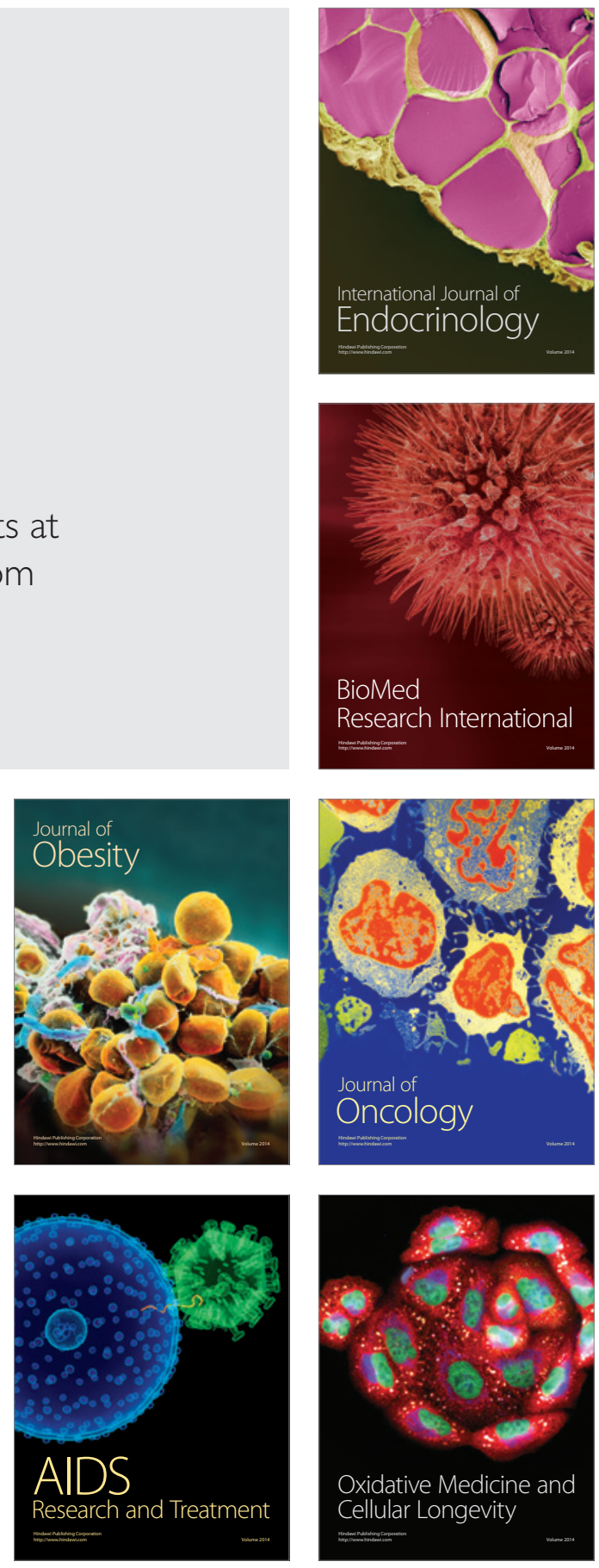